\title{
Developmental Regulation of the Proteolysis of the p35 Cyclin-Dependent Kinase 5 Activator by Phosphorylation
}

\author{
Taro Saito, ${ }^{1}$ Reiko Onuki, ${ }^{1}$ Yuichi Fujita, ${ }^{1}$ Gen-ichi Kusakawa, ${ }^{1,2}$ Koichi Ishiguro, ${ }^{3}$ James A. Bibb, ${ }^{4}$ Takeo Kishimoto, ${ }^{2}$ \\ and Shin-ichi Hisanaga ${ }^{1}$ \\ ${ }^{1}$ Department of Biological Sciences, Graduate School of Science, Tokyo Metropolitan University, Minami-Osawa, Hachiohji, Tokyo 192-0397, Japan, \\ ${ }^{2}$ Laboratory of Cell and Developmental Biology, Department of Biological Information, Graduate School of Bioscience and Biotechnology, Tokyo Institute of \\ Technology, Yokohama 226-8501, Japan, ${ }^{3}$ Mitsubishi Kasei Institute of Life Sciences, Machida, Tokyo 194-8511, Japan, and ${ }^{4}$ Department of Psychiatry, \\ University of Texas Southwestern Medical Center, Dallas, Texas 75390
}

Cyclin-dependent kinase 5 (Cdk5), a cdc2-related kinase expressed in postmitotic neurons, is activated by association with a brainspecific activator, $\mathrm{p} 35$. It has been suggested that the conversion of $\mathrm{p} 35$ to $\mathrm{p} 25$ by the protease calpain is involved in neuronal cell death. However, p35 protein is turned over rapidly via proteasomal degradation in living neurons. In this study we show that the phosphorylation of $\mathrm{p} 35$ by Cdk5 suppresses the cleavage to 25 by calpain, whereas phosphorylation facilitates the proteasomal degradation of $\mathrm{p} 35$. The phosphorylation site in $\mathrm{p} 35$ that might be involved in preventing calpain cleavage was distinct from the phosphorylation site involved in facilitating proteasomal degradation. A phosphorylated form of $\mathrm{p} 35$ that was resistant to cleavage by calpain was more prevalent in the fetal brain, whereas the unphosphorylated form of p35 occurred in the adult brain. These results suggest that the phosphorylation of p35 serves as a protective mechanism that suppresses the generation of $\mathrm{p} 25$ in developing brains.

Key words: calpain; Cdk5; neuron; proteasome; protein kinase; cell death; development

\section{Introduction}

Cyclin-dependent kinase $5(\mathrm{Cdk} 5)$ is a unique member of the Cdk family whose activity has been detected primarily in postmitotic neurons. The Cdk5 activator p35, as well as its isoform p39, is expressed only in neurons (Lew et al., 1994; Tsai et al., 1994). Mice lacking p 35 or C dk 5 display an altered pattern of lamination in the cerebral cortex, with an inverse packing order of postmitotic cortical neurons (Ohshima et al., 1996; Chae et al., 1997), suggesting that $\mathrm{Cdk} 5 / \mathrm{p} 35$ is involved in the migratory behavior of postmitotic neurons during brain development.

Cdk5 also has been implicated in the pathological degeneration of neurons. It has been suggested that dysregulation of Cdk5 causes the hyperphosphorylation of tau, thereby contributing to neurofibrillary tangle formation (Ishiguro et al., 1992a; Baumann et al., 1993). Cleavage of p35 to p 25 was shown to be catalyzed by calpain (Kusakawa et al., 2000; Lee et al., 2000), a protease that is involved in various types of cell death (Wang, 2000). Accumulation of p25 has been observed in cultured neurons undergoing cell death (Kusakawa et al., 2000; Lee et al., 2000) and in the brains of Alzheimer's patients (Patrick et al., 1999). Cleavage of p35 to p25 changed the subcellular distribution of active Cdk5 from the particulate to the cytosolic fraction (Kusakawa et al., 2000; Lee et al., 2000), thereby allowing potentially aberrant phosphorylation of neuronal proteins and adversely affecting the

Received June 21, 2002; revised Dec. 4, 2002; accepted Dec. 6, 2002

This work was supported in part by the Naito Foundation (S.H.) and by grants-in-aid from the Ministry of Education, Science and Culture of Japan (S.H., T.K.) and the Core Research for Evolutional Science and Technology of the Japan Science and Technology Corporation (T.K.). We thank Dr. L.-H. Tsai (Harvard Medical School) for providing cDNAs of human Cdk5 and $\mathrm{p} 35$.

Correspondence should be addressed to Taro Saito, Department of Biological Sciences, Graduate School of Science, Tokyo Metropolitan University, Minami-Osawa, Hachiohji, Tokyo 192-0397, Japan. E-mail: tasaito@comp.metro-u.ac.jp. Copyright $\odot$ 2003 Society for Neuroscience $\quad 0270-6474 / 03 / 231189-09 \$ 15.00 / 0$ survival of postmitotic neurons. Therefore, it would be useful to identify the mechanisms involved in regulating the stability of p35 and/or its conversion to p25.

Cdk5 is activated when associated with p35 or its isoform, p39 (Lee et al., 1996; Poon et al., 1997). The total amount of available activators appears to be a major determinant of Cdk5 activity. p35 is a short-lived protein that is degraded by proteasomes in primary cultured neurons (Patrick et al., 1998; Saito et al., 1998), and the degradation of p35 might be a critical regulator of its levels. It has been suggested that proteasomal degradation of p35 is promoted by phosphorylation (Patrick et al., 1998; Saito et al., 1998), although the role of phosphorylation in the degradation of p35 in neurons has not been demonstrated.

In this study we show that the susceptibility of p35 to calpaindependent cleavage versus proteasomal degradation is different in embryonic and adult brains. The phosphorylation state of p35 also changes during the process of development. Phosphorylated p35 predominates in fetal rat brains and is particularly resistant to cleavage by calpain, but it is susceptible to proteasomal degradation. However, unphosphorylated p35 predominates in adult rat brains, where it is resistant to proteasomal degradation but is readily cleaved by calpain to $\mathrm{p} 25$. These data suggest that phosphorylation suppresses cleavage to $\mathrm{p} 25$ by calpain and targets $\mathrm{p} 35$ to degradation by proteasomes, and the data suggest that this regulatory mechanism is controlled in a developmental manner.

\section{Materials and Methods}

Materials. Okadaic acid and bacterial alkaline phosphatase were purchased from Wako Chemicals (Osaka, Japan), lactacystin and benzyloxycarbonyl-leucyl-leucyl-leucinal (MG132) were from Calbiochem (San Diego, CA), and skeletal muscle m-calpain and casein were from Sigma (St. Louis, MO). Cyclosporin A was provided by Drs. Takao Kataoka and Kazuo Nagai (Tokyo Institute of Technology, Tokyo, 
Japan). Butyrolactone-I (BL-I) was provided by Dr. Akira Okuyama (Banyu Pharmaceutical Research Institute in collaboration with Merck Research Laboratories, Tsukuba, Japan) (Hisanaga et al., 1993). The antip35 antibody C-19 and the anti-Cdk5 antibody C-8 were obtained from Santa Cruz Biotechnology (Santa Cruz, CA). The anti-Cdk5 antibody DC17 was obtained from Calbiochem. The anti- $\alpha$-spectrin monoclonal antibody 1622 was obtained from Chemicon (Temecula, CA). The monoclonal anti-FLAG antibody M2 was obtained from Sigma. An antibody against the $\mathrm{N}$ terminus of bovine $\mathrm{p} 23$ (p23N), a truncated form of p35, has been described previously (Uchida et al., 1994).

Preparation of cerebral cortical slices. Cerebral cortical slices $(200 \mu \mathrm{m}$ thick) were prepared from fetal [embryonic day 18 (E18)] or adult (week 15) rat brains using a tissue chopper and were incubated in Krebs' buffer (in mM: $124 \mathrm{NaCl}, 4 \mathrm{KCl}, 26 \mathrm{NaHCO}_{3}, 1.5 \mathrm{CaCl}_{2}, 1.25 \mathrm{KH}_{2} \mathrm{PO}_{4}, 1.5$ $\mathrm{MgSO}_{4}$, and $10 \mathrm{D}$-glucose, $\mathrm{pH} 7.4$ ) at $37^{\circ} \mathrm{C}$ under $5 \% \mathrm{CO}_{2}$. After incubation for $3 \mathrm{hr}$, slices were treated with $10 \mu \mathrm{M}$ cycloheximide (CHX) for 3 hr. Slices were rapidly lysed in lysis buffer (31.25 mm Tris-HCl, pH 6.8, $1 \%$ SDS, $5 \%$ glycerol, and $2.5 \% \beta$-mercaptoethanol) by sonication, followed by boiling for $5 \mathrm{~min}$. The proteins p 35 and p 25 were detected by immunoblotting with anti-p35 (p23N) antibody.

Cell culture and metabolic phosphorylation of $p 35$. Cerebral cortical neurons were prepared from 17-d-old embryonic rat brains as described previously (Tokuoka et al., 2000). For metabolic phosphorylation of p35, neurons were cultured in the presence of $\left[{ }^{32} \mathrm{P}\right]$ orthophosphate in phosphate-free DMEM for $4 \mathrm{hr}$. To induce hyperphosphorylation of $\mathrm{p} 35$, $0.1 \mu \mathrm{M}$ okadaic acid was added to the medium for the final $2 \mathrm{hr}$ of the $4 \mathrm{hr}$ labeling period. MG132 $(50 \mu \mathrm{M})$ was added $1 \mathrm{hr}$ before the addition of okadaic acid to suppress the proteasome-dependent degradation of $\mathrm{p} 35$. Neurons were suspended in RIPA buffer [20 mM Tris-HCl, pH 7.5, $1 \mathrm{~mm}$ EGTA, 1 mm EDTA, $0.15 \mathrm{~m} \mathrm{NaCl}, 1 \%$ Nonidet P-40, $0.1 \%$ SDS, $0.1 \%$ sodium deoxycholate, $10 \mathrm{~mm} \beta$-glycerophosphate, $5 \mathrm{~mm} \mathrm{NaF}, 1 \mathrm{~mm}$ p-nitrophosphate, 0.2 mm Pefabloc SC (Merck, Darmstadt, Germany), 1 $\mu \mathrm{g} / \mathrm{ml}$ leupeptin, $100 \mu \mathrm{M}$ E64, and $1 \mathrm{~mm}$ dithiothreitol (DTT)] and lysed by freezing and thawing. The extract was prepared as a supernatant by centrifugation at $10,000 \times g$ for $10 \mathrm{~min}$. p35 was isolated from the extract by immunoprecipitation with C-19 as described previously (Kusakawa et al., 2000). ${ }^{32} \mathrm{P}$ incorporation into $\mathrm{p} 35$ was detected by a BAS 2000 image analyzer (Fuji Film, Tokyo, Japan) after SDS-PAGE.

Preparation of the cultured neuron extract or rat brain extracts and in vitro phosphorylation of $p 35$. Cultured neurons and fetal or adult rat brains were homogenized with HEPES buffer (in mM: 20 HEPES, pH 7.5, $5 \mathrm{KCl}, 1.5 \mathrm{MgCl}_{2}, 1 \mathrm{EGTA}$, and $1 \mathrm{DTT}$ ) in a Teflon pestle homogenizer and centrifuged at $10,000 \times g$ for $15 \mathrm{~min}$ to collect the extract as a supernatant (Saito et al., 1998). The extracts of cultured neurons and brain were incubated in the absence or presence of $1 \mathrm{~mm} \mathrm{ATP} \mathrm{at} 30^{\circ} \mathrm{C}$ for the indicated times. p35 and Cdk5 were detected by immunoblotting with anti-p35 (p23N) and anti-Cdk5 (DC17) antibodies, respectively, after Tris/tricine SDS-PAGE.

p35 in brain extracts was phosphorylated by incubation at $30^{\circ} \mathrm{C}$ in the presence of $1 \mathrm{mM} \mathrm{MgCl}_{2}$ and $0.1 \mathrm{~mm}$ ATP containing $\left[\gamma^{-}{ }^{32} \mathrm{P}\right]$ ATP and isolated by immunoprecipitation with C-19. Phosphorylation was analyzed with an image analyzer after SDS-PAGE.

Rat cerebral cortices of various ages were homogenized in $10 \mathrm{vol}$ of 3-morpholinopropanesulfonic acid (MOPS) buffer (20 mM MOPS, $\mathrm{pH}$ 6.8, 1 m EGTA, 0.1 mm EDTA, $0.3 \mathrm{M} \mathrm{NaCl}, 1 \mathrm{~mm} \mathrm{MgCl}_{2}, 0.5 \%$ Nonidet P-40, $10 \mathrm{~mm} \beta$-glycerophosphate, $5 \mathrm{~mm} \mathrm{NaF}, 0.2 \mathrm{~mm}$ Pefabloc SC, 1 $\mu \mathrm{g} / \mathrm{ml}$ leupeptin, and $1 \mathrm{~mm}$ DTT) with a Teflon pestle homogenizer and centrifuged at $10,000 \times g$ for 15 min to collect the extract as a supernatant.

Cdk5 kinase activity assay. Cdk5 bound to phosphorylated or unphosphorylated p35 was produced by incubating fetal or adult brain extracts in the presence or absence of $1 \mathrm{~mm} \mathrm{ATP}$ at $37^{\circ} \mathrm{C}$ for $1 \mathrm{hr}$. The kinase activity of Cdk5 immunoprecipitated with anti-Cdk5 antibody (C-8) was measured at $37^{\circ} \mathrm{C}$ using histone $\mathrm{H} 1$ or bacterially expressed human tau as substrates (Kusakawa et al., 2000). An expression vector encoding the longest isoform of human tau (htau40) was provided by Dr. M. Goedert (Medical Research Council, Oxford, UK). Tau was purified from the heat-stable supernatant of the Escherichia coli lysate by phosphocellulose column chromatography.
In vitro translation of $p 35$ in reticulocyte lysate and reconstitution with Cdk5. cDNAs of human Cdk5 and p35 in pCMV were provided by Dr. L.-H. Tsai (Harvard Medical School, Boston, MA) (Tsai et al., 1994). p35 cDNA was amplified by PCR using oligonucleotides 5 '-GGCGAATTCATGGGCACGGTGCTGTCCCT-3' and 5'-GGCCTCGAGTCACCGATCCAGGCCTAGGA-3' as primers. The PCR product was cleaved with EcoRI and $\mathrm{XhoI}$ and ligated into pET23a (Novagen, Madison, WI), an expression vector for protein $6 \mathrm{xHis}$ tagged at the $\mathrm{C}$-terminal end. $\mathrm{p} 35$ was translated in vitro with a TNT/T7 Rabbit Reticulocyte Lysate Kit (Promega, Madison, WI) using pET23a-p35 in the presence of $\left[{ }^{35} \mathrm{~S}\right]$ methionine for $1 \mathrm{hr}$ at $30^{\circ} \mathrm{C}$ (total reaction, $50 \mu \mathrm{l}$ ) according to the manufacturer's protocol. A mutant $\mathrm{p} 35$, designated $\mathrm{p} 35^{4 \mathrm{~A}}$, in which $\mathrm{Ser}^{8}, \mathrm{Thr}^{138}, \mathrm{Ser}^{170}$, and $\mathrm{Thr}^{197}$ were replaced with Ala, was generated by site-directed mutagenesis using pET23a-p35 as a template.

${ }^{35}$ S-labeled p35 expressed in reticulocyte lysate was incubated with rat brain extract at $30^{\circ} \mathrm{C}$ for $1 \mathrm{hr}$ to form a complex with Cdk5. The p35/ Cdk5 complex was immunoprecipitated with anti-Cdk5 antibody (C-8) bound to protein A-Sepharose CL-4B (Amersham Biosciences, Uppsala, Sweden). The $\mathrm{p} 35 / \mathrm{Cdk} 5$ complex was incubated in the presence of $1 \mathrm{~mm}$ $\mathrm{Mg}$-ATP at $30^{\circ} \mathrm{C}$. The reaction was stopped by the addition of SDS-PAGE sample buffer, and p 35 was detected with a BAS 2000 image analyzer after Tris/tricine SDS-PAGE.

Preparation of recombinant $p 35 / C d k 5$ with a baculovirus expression system. cDNA for human Cdk5 in pCMV was excised with BamHI and ligated into the BamHI site of a baculovirus transfer vector, BacPAK9 (Clontech, Palo Alto, CA). Kinase-dead Cdk5 ( Lys $^{33}$ replaced with Thr) was generated by site-directed mutagenesis (Nikolic et al., 1996). Amplification by PCR of $6 \mathrm{xHis}$-tagged $\mathrm{p} 35$ or $\mathrm{p} 35^{4 \mathrm{~A}}$ was performed using pET23a-p35 or pET23a-p35 ${ }^{4 \mathrm{~A}}$ as a template, respectively, and the oligonucleotides 5'-GGCGAATTCATGGGCACGGTGCTGTCCCT-3' and 5'GGGCTTTAGCGGCCGCCGGATCTCAGTG- $3^{\prime}$ as primers. The PCR product was cleaved with EcoRI and NotI and ligated into BacPAK9. BacPAK9, containing either Cdk5 or p35 cDNA, was cotransfected with BacPAK6 virus DNA (Clontech) into Sf9 cells according to the manufacturer's instructions. The p35/Cdk5 complex was purified from an Sf9 cell extract with nickel beads (Ni-NTA; Qiagen, Hilden, Germany).

Adenoviral expression of $p 35$ in cultured neurons. FLAG-tagged wildtype p35 or mutant $\mathrm{p} 35^{4 \mathrm{~A}}$ was amplified by PCR using pET23a-p35 or pET23a-p $35^{4 \mathrm{~A}}$ as a template, respectively; oligonucleotides $5^{\prime}$-GCTCTAGAATGGGCACGGTGCTGTCCCTGTCTCCCAGC-3' and 5'-GGGGTACCTCACTTGTCGTCATCGTCTTTGTAGTCCCGATCCAGGCCTAGG-3' were used as primers for pET23a-p35, and oligonucleotides 5'-GCTCTAGAATGGGCACGGTGCTGTCCCTGGCTCCCAGC-3' and 5'-GGGGTACCTCACTTGTCGTCATCGTCTTTGTAGTCCCGATCCAGGCCTAGG-3' were used as primers for pET23a-p35 ${ }^{4 \mathrm{~A}}$. The PCR products were cleaved with $K p n \mathrm{I}$ and $\mathrm{Xba \textrm {I }}$ and ligated into pShuttle (Clontech). p35-FLAG or p $35^{4 \mathrm{~A}}$-FLAG cDNA was ligated into Adeno-X viral DNA (Clontech), and adenoviruses were generated according to the manufacturer's instructions. Embryonic cultured neurons were infected $4 \mathrm{~d}$ after plating at a multiplicity of infection of five and were used after $2 \mathrm{~d}$.

Calpain activity assay. Casein $(0.25 \mathrm{mg} / \mathrm{ml})$ was incubated with fetal or adult brain extract at equal protein concentrations in the presence or absence of $5 \mathrm{~mm} \mathrm{CaCl}_{2}$ at $37^{\circ} \mathrm{C}$ for $1 \mathrm{hr}$. The breakdown of casein was estimated by densitometric scanning of Coomassie brilliant blue-stained SDS-PAGE gels. p35 immunoprecipitated from the adult brain extract was cleaved to p 25 by incubation with the fetal or adult brain extracts in the presence of $5 \mathrm{~mm} \mathrm{CaCl}_{2}$ for 5 and $10 \mathrm{~min}$. p35 and p25 were detected by immunoblotting with $\mathrm{C}-19$.

Degradation of $p 35$ by proteasomes and cleavage of $p 35$ to $p 25$ by calpain in vitro. Degradation of $\mathrm{p} 35$ by proteasomes was induced by incubation of the extracts of cultured neurons and brains with $1 \mathrm{~mm}$ ATP and $10 \mu \mathrm{M}$ okadaic acid at $30^{\circ} \mathrm{C}$ for $1-3 \mathrm{hr}$. p35 in the rat brain extract was cleaved to p25 by incubation with $5 \mathrm{~mm} \mathrm{CaCl}_{2}$ at $30^{\circ} \mathrm{C}$. p 35 and p 25 were detected by immunoblotting with anti-p35 (p23N) antibody, and Cdk5 was detected with DC-17 antibody, after 10\% Tris/tricine SDS-PAGE. p35, which was translated in reticulocyte lysate and then reconstituted with $\mathrm{Cdk} 5$, was cleaved to $\mathrm{p} 25$ by purified $\mathrm{m}$-calpain $(0.04 \mathrm{U} / \mathrm{ml})$ in the presence of $1 \mathrm{mM} \mathrm{CaCl}_{2}$. 


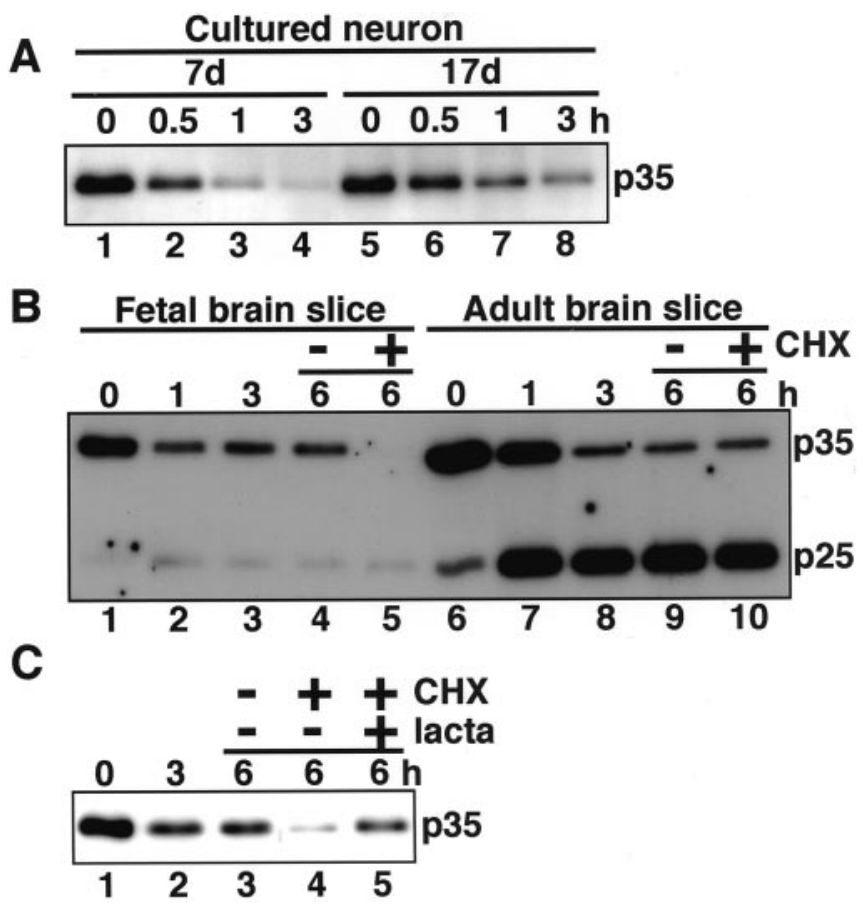

Figure 1. Changes in the susceptibility of $\mathrm{p} 35$ to proteasomal degradation and calpain cleavage in cultured neurons and brain slices. $A$, Turnover rate of $\mathrm{p} 35$ in neurons cultured for different periods. Rat brain cortical neurons cultured for $7 \mathrm{~d}$ (Ianes 1-4) or $17 \mathrm{~d}$ (Ianes 5-8) after plating were treated with $10 \mu \mathrm{g} / \mathrm{ml} \mathrm{CHX} \mathrm{for} 0 \mathrm{hr}$ (lanes 1 and 5), $0.5 \mathrm{hr}$ (lanes 2 and 6 ), $1 \mathrm{hr}$ (lanes 3 and 7), or $3 \mathrm{hr}$ (lanes 4 and 8 ). The levels of $\mathrm{p} 35$ were examined by immunoblotting. B, Proteolysis of p35 in cultured brain slices. Cortical slices from rat brains at E18 (lanes 1-5) or postnatal week 15 (lanes $6-10$ ) were cultured in Krebs' buffer for $0 \mathrm{hr}$ (lanes 1 and 6 ), $1 \mathrm{hr}$ (lanes 2 and 7 ), $3 \mathrm{hr}$ (lanes 3 and 8), or $6 \mathrm{hr}$ (lanes 4, 5, 9, and 10). The levels of p35 and p25 were examined by immunoblotting. After incubating slice preparations for $3 \mathrm{hr}$, we examined the effects of 10 $\mu \mathrm{g} / \mathrm{ml} \mathrm{CHX}$ on p35 levels during an additional $3 \mathrm{hr}$ incubation (lanes 5 and 10). C, Effect of lactacystin (lacta) on degradation of $\mathrm{p} 35$ in brain slices. Fetal rat brain slices were cultured for 3 $\mathrm{hr}$ (lane 2) or $6 \mathrm{hr}$ (lanes 3-5) after slice preparation. Three hours after slice preparation, 10 $\mu \mathrm{g} / \mathrm{ml} \mathrm{CHX}$ alone (lane 4) or both CHX and $20 \mu \mathrm{m}$ lactacystin (lane 5) were added for an additional $3 \mathrm{hr}$, and the levels of $\mathrm{p} 35$ were examined by immunoblotting.

SDS-PAGE, immunoblotting, and determination of protein concentrations. SDS-PAGE was performed according to the method of Laemmli (1970) using a 12.5\% polyacrylamide gel. Tris/tricine SDS-PAGE with a $10 \%$ polyacrylamide gel was used to separate phosphorylated and unphosphorylated p35 (Schagger and von Jagow, 1987; Ploug et al., 1989). Proteins were transferred to polyvinylidene difluoride membrane (Millipore, Bedford, MA), reacted with primary antibodies, and then reacted with peroxidase-conjugated anti-rabbit or anti-mouse IgG secondary antibodies (Dako, Glostrup, Denmark). The reaction was detected with the enhanced chemiluminescence system (Amersham Biosciences). Protein concentrations were determined with Coomassie protein assay reagent (Pierce, Rockford, IL).

\section{Results}

p35 proteolytic patterns differ in fetal and adult rats

In cultured neurons, p35 exhibits a short half-life (Patrick et al., 1998; Saito et al., 1998). However, the half-life is extended when the cultivation period is extended (Fig. $1 \mathrm{~A}$ ). The protein synthesis inhibitor CHX was added at $10 \mu \mathrm{g} / \mathrm{ml}$ to the culture medium on day 7 or day 17 after plating to estimate the half-life of p35. The rate of decrease of $\mathrm{p} 35$ was attenuated in cortical neurons cultured for $17 \mathrm{~d}$ (Fig. $1 A, 17 d$ ). This result suggests that an alteration in p35 turnover is associated with the maturation of neurons.

To assess this possibility further, we compared the turnover of p35 in embryonic and adult rat brains. We used slice cultures instead of cultured neurons, because it is difficult to prepare cultured neurons from adult rat brains. Cerebral cortical slices were prepared from fetal (E18) or adult (postnatal week 15) rat brains, and the turnover of p35 was assessed by immunoblotting. However, substantial amounts of p35 were cleaved to p25 in cortical slices from adult brains (Fig. $1 B$, Adult brain slice). p25 had accumulated $1 \mathrm{hr}$ after slice preparation (Fig. $1 B$, lane 7 ), whereas p35 levels were substantially reduced by $3 \mathrm{hr}$ of incubation (Fig. $1 B$, lane 8 ) and remained unchanged for at least the next $3 \mathrm{hr}$ (Fig. $1 B$, lane 9). Trypan blue staining revealed substantial levels of dead neurons in slices from adult brains (data not shown). It is possible that cleavage of p 35 to p 25 was induced in these dead neurons. In contrast, the generation of p25 was almost absent in fetal brain slices incubated for $0-6 \mathrm{hr}$ at $37^{\circ} \mathrm{C}$ (Fig. $1 \mathrm{~B}$, lanes $1-4$ ), although the number of viable neurons also decreased (data not shown), and the amount of p35 decreased during the first hour of culture (Fig. $1 B$, lane 2).

The turnover of the p35 that remained after $3 \mathrm{hr}$ of incubation was assessed next. After slice preparation and a $3 \mathrm{hr}$ incubation period, slices were incubated for an additional $3 \mathrm{hr}$ in the presence of $\mathrm{CHX}$, which resulted in a reduction of p35 to undetectable levels in fetal slice cultures (Fig. $1 \mathrm{~B}$, lane 5). This effect was attenuated significantly by the addition to the culture medium of lactacystin $(20 \mu \mathrm{M})$, a specific inhibitor of proteasomes (Fig. 1C, lanes 4 and 5). In contrast, p35 levels in adult brain slices were not affected by the presence of CHX (Fig. $1 \mathrm{~B}$, lanes 9 and 10). This effect in adult brain slices was not attributable to insufficient penetration by CHX. The inhibition of protein synthesis in the adult brain slices by CHX was confirmed by the suppression of $\left[{ }^{35} \mathrm{~S}\right]$ methionine incorporation (data not shown). Therefore, $\mathrm{p} 35$ was turned over more rapidly via proteasomal degradation in the short-term cultured neurons and fetal brain slices than in the long-term cultured neurons and adult brain slices. These results suggest that the susceptibility to calpain cleavage and the half-life of p35 change with neuronal maturation.

\section{The phosphorylation status of p35 changes during rat brain development}

The generation of $\mathrm{p} 25$ in adult cortical slice cultures was probably attributable to calpain activation induced by injuries during the preparation of brain slices. However, p35 in fetal brain slices did not undergo cleavage to $\mathrm{p} 25$, although similar damage could have occurred to embryonic neurons. Calpain activity was measured in fetal and adult brain extracts using as substrate either casein or p35 immunoprecipitated from adult brain extracts. It is interesting to note that the calpain activity toward both substrates was even greater in the brain extract prepared from fetal tissue than in that prepared from adult tissue (Fig. $2 A, B$ ). In addition, the activation of calpain was shown in fetal brain slices as well as in adult brain slices by detection of the calpain-specific breakdown product of $\alpha$-spectrin (Saido et al., 1993) (data not shown). Therefore, increased calpain activity in adult brain tissue might not be the cause of the differences in the levels of p 25 generated in fetal and adult brain slices.

We have reported previously that cultured neurons from embryonic brains display calpain-dependent cleavage of p35 to p25 when treated with $\mathrm{Ca}^{2+}$ ionophore (A23187) (Kusakawa et al., 2000). In an attempt to determine why p35 is cleaved to p25 in cultured neurons as well as in adult brain slices, the effects of cyclosporin A, a protein phosphatase $2 \mathrm{~B}$ ( $\mathrm{PP} 2 \mathrm{~B}$ ) inhibitor, on $\mathrm{Ca}^{2+}$ ionophore-induced cleavage of p35 to p25 in embryonic cultured neurons was assessed (Fig. 2C, $+C y s A$ ). Cleavage of p35 to $\mathrm{p} 25$ in response to the addition of $5 \mu \mathrm{M}$ A23187 was attenuated 


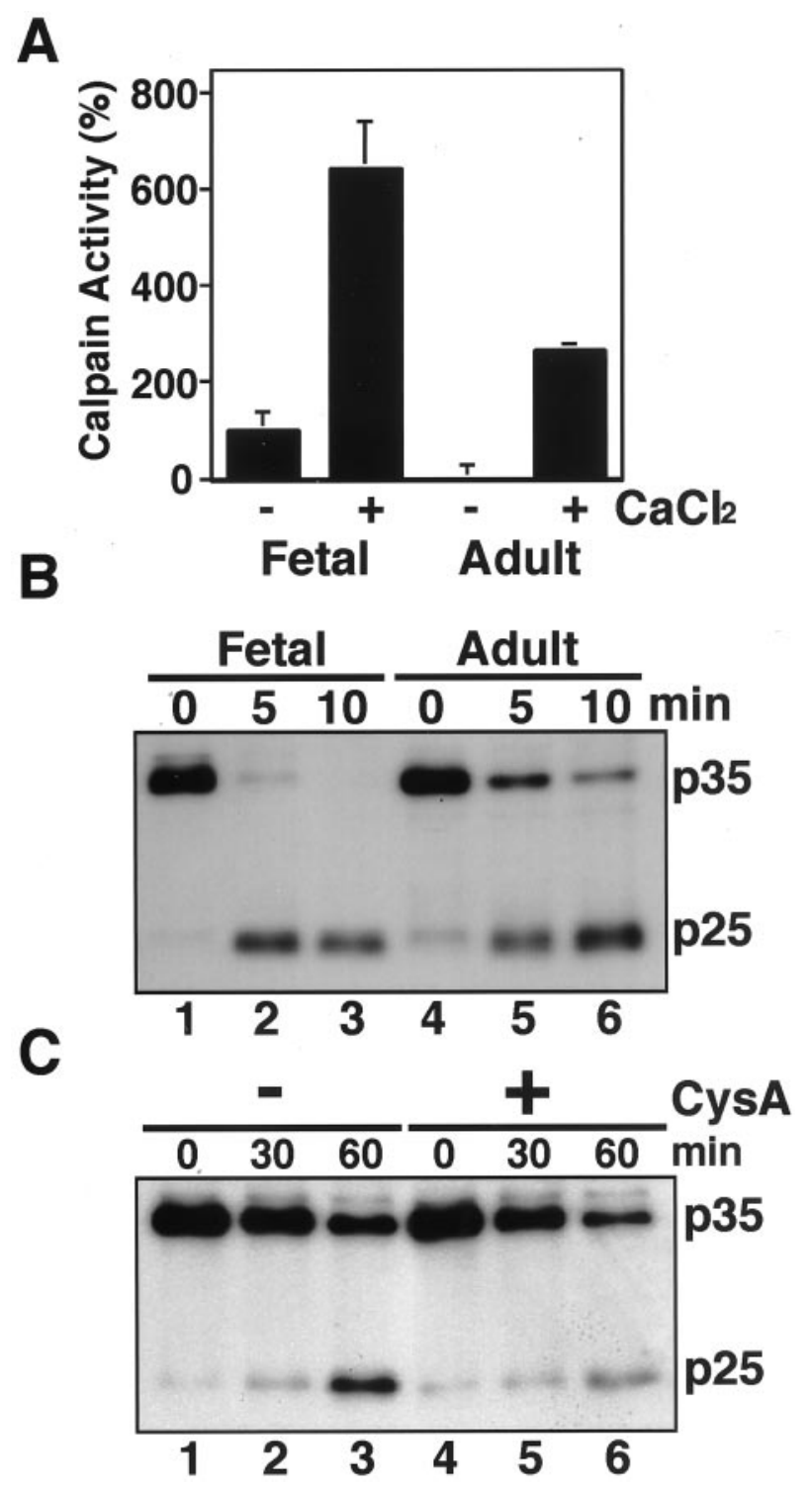

Figure 2. Calpain activity in fetal and adult brain extract and the effect of cyclosporin A on calpain cleavage of $\mathrm{p} 35$ in $\mathrm{Ca}^{2+}$ ionophore-treated neurons. A, Calpain activity in fetal and adult brain extracts was measured using casein as a substrate. Data are means \pm SE from measurements made in triplicate. $B$, Calpain activity in fetal and adult brain extracts against p35 immunoprecipitated from adult brain extract. Immunoprecipitated p 35 was incubated with 5 $\mathrm{mm} \mathrm{CaCl} 2$ in fetal (lanes 1-3) or adult (lanes 4 - 6) brain extracts for 0 min (lanes 1 and 4), 5 min (lanes 2 and 5), and 10 min (lanes 3 and 6 ) and immunoblotted for p35 and p25. C, PP2B is involved in the $\mathrm{Ca}^{2+}$ ionophore-induced cleavage of $\mathrm{p} 35$ in embryonic cultured neurons. Embryonic cortical neurons cultured for $7 \mathrm{~d}$ were treated with both $5 \mu \mathrm{m} \mathrm{A23187} \mathrm{and} 5 \mathrm{~mm} \mathrm{CaCl}$ in the absence (lanes 1-3) or presence (lanes 4-6) of $2 \mu \mathrm{m}$ cyclosporin A (CysA) for 0 min (lanes 1 and 4), 30 min (lanes 2 and 5), or 60 min (lanes 3 and 6). p35 and p25 were detected by immunoblotting.

by the addition of cyclosporin A, indicating that $\mathrm{PP} 2 \mathrm{~B}$ activation can promote cleavage of $\mathrm{p} 35$ to $\mathrm{p} 25$. Conversely, treatment of the embryonic slices with $\mathrm{Ca}^{2+}$ ionophore induced the generation of p25 (data not shown). Therefore, slice preparation and $\mathrm{Ca}^{2+}$ ionophore treatment may activate differently the $\mathrm{Ca}^{2+}$ dependent enzymes calpain and PP2B.

These results suggest that phosphorylation might be associated with the cleavage of $\mathrm{p} 35$ to $\mathrm{p} 25$, and that the phosphorylation status of p35 may be different in fetal and adult brains. To examine this possibility, homogenates of acutely dissected whole brain
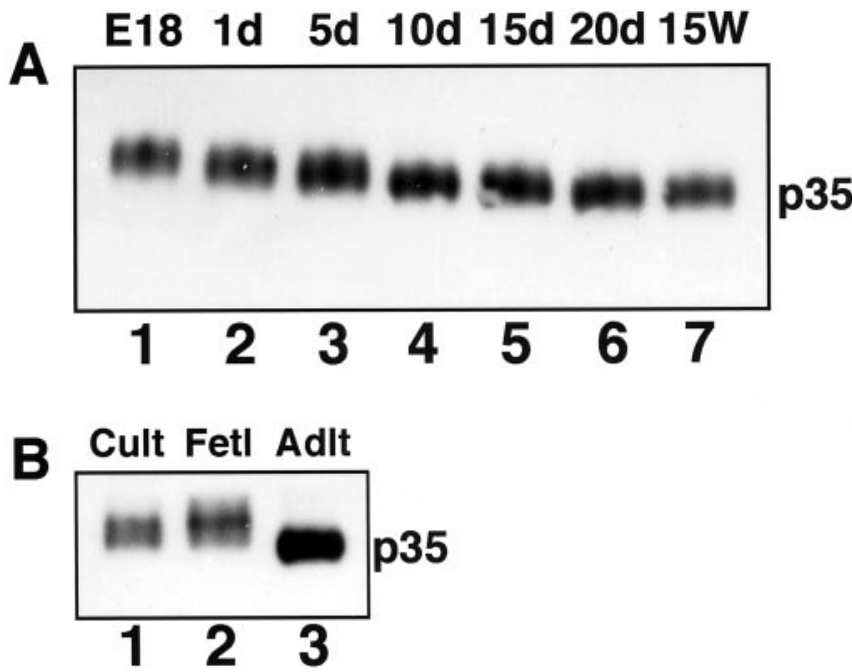

Figure 3. Electrophoretic mobility of $\mathrm{p} 35$ at various stages of brain development. $A$, Brains were prepared from 18-d-old rat embryos (Iane 1); rats at postnatal day 1 (Iane 2), day 5 (Iane 3), day 10 (lane 4), day 15 (lane 5), and day 20 (lane 6); and 15-week-old rats (lane 7). Ten micrograms of proteins were subjected to Tris/tricine SDS-PAGE. Immunoblots of rat brain cortical homogenates show the electrophoretic mobility change of $p 35 . B$, Immunoblot of $p 35$ in homogenates of cultured neurons (Cult; lane 1) and fetal (Fett; /ane 2) and adult (Adlt; lane 3) rat brain cortices subjected to Tris/tricine SDS-PAGE.

at different stages of development were immunoblotted for p35 (Fig. 3A). However, p35 levels were unchanged at all developmental stages examined, an increase in the electrophoretic mobility of p35 was observed as brain development proceeded, p35 from embryonic brains migrated more slowly, and p35 from adult brains migrated more rapidly (Fig. $3 A$, lanes 1 and 7 ). p35 from cultured neurons exhibited a slower mobility (Fig. 3B, lane 1) than that of p35 from adult brain tissue (Fig. 3B, lane 3) but migrated faster than p 35 from fetal brain homogenates (Fig. $3 B$, lane 2).

To determine whether these developmentally regulated electrophoretic mobility changes of p35 are attributable to phosphorylation, extracts from cortical cultures, fetal brain, or adult brain were incubated in the absence or presence of ATP (Fig. 4A). When cultured neuronal extracts were incubated without ATP, the electrophoretic mobility of p35 on Tris/tricine gels was increased (Fig. 4A, lanes 1-3). Okadaic acid, a Ser/Thr protein phosphatase inhibitor, prevented this mobility change (data not shown). When ATP was added to the extract, there was no change in the electrophoretic mobility of p35 (Fig. 4A, lanes 4 and 5). A similar increase in electrophoretic mobility was observed with the fetal brain extract, in an ATP-dependent manner (Fig. 4A, lanes $6-10)$. In contrast, the mobility of $\mathrm{p} 35$ from the adult rat brain extract did not change in the absence of ATP (Fig. $4 A$, lanes 11-13). When ATP was added, the mobility of p35 increased (Fig. $4 A$, lanes 14 and 15 ) to the position observed for the other extracts. No electrophoretic mobility change was observed in Cdk5 as a result of the incubation of any of the extracts in the absence or presence of ATP (Fig. 4A, bottom). In addition, treatment with bacterial alkaline phosphatase induced an increase in electrophoretic mobility of p35 immunoprecipitated from fetal brain extracts (Fig. 4 B, lane 2). These results suggest that the changes in electrophoretic mobility of p 35 were dependent on its phosphorylation state(s). Whereas p35 from either cultured neurons or fetal brain extracts was phosphorylated, p35 from adult brain extracts was unphosphorylated.

To demonstrate more clearly that p35 occurs as a phospho- 
A

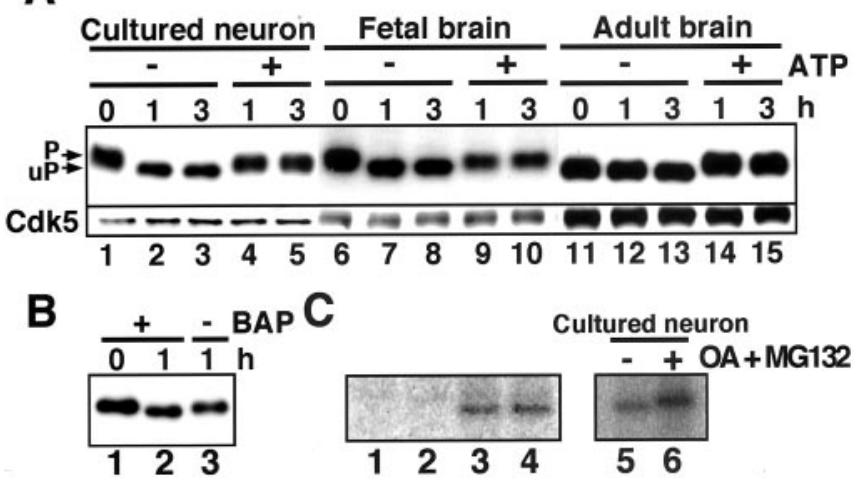

Figure 4. Phosphorylation of $\mathrm{p} 35$ in extracts from cultured neurons, extracts from fetal and adult brains, and in cultured neurons. $A$, Phosphorylation-dependent electrophoretic mobility change of $\mathrm{p} 35$. Extracts prepared from cultured neurons (lanes 1-5) and fetal (Ianes 6-10) and adult (lanes 11-15) brains were incubated in the absence (lanes 1-3, 6-8, and 11-13) or presence (lanes 4, 5, 9, 10, 14, and 15) of $1 \mathrm{~mm}$ ATP for $0 \mathrm{hr}$ (lanes 1, 6, and 11), $1 \mathrm{hr}$ (lanes 2, 4, 7, 9, 12, and 14), or $3 \mathrm{hr}$ (lanes 3, 5, 8, 10, 13, and 15) and immunoblotted for p35. The positions of the phosphorylated and unphosphorylated forms of p 35 are indicated by $P$ and $u P$, respectively. Western blots of the same samples probed for $C \mathrm{dk} 5$ are shown at the bottom. $B$, Electrophoretic mobility change of p35 induced by treatment with bacterial alkaline phosphatase (BAP). p35 immunoprecipitated from fetal brain extract was incubated in the absence (lane 3) or presence (lane 2) of BAP for $1 \mathrm{hr}$. p35 was detected by immunoblotting. C, The phosphorylation of p35 in fetal (lanes 1 and 3) or adult (lanes 2 and 4 ) brain extracts in vitro and in fetal rat brain cortical neurons in situ (lanes 5 and 6). Phosphorylation signal was detected with a molecular weight corresponding to $35 \mathrm{kDa}$ when fetal (lane 3) or adult (lane 4) brain extracts were incubated for $1 \mathrm{hr}$ in the presence of [ $\boldsymbol{\gamma}^{32}$ P]ATP followed by immunoprecipitation with anti-p35 antibody. Control immunoprecipitations with rabbit lgG are shown in lane 1 for the fetal brain extract and lane 2 for the adult brain extract. Phosphorylation of $p 35$ was observed when p35 was immunoprecipitated from extracts of neurons that were prelabeled with [ ${ }^{32}$ P]orthophosphate (lane 5). The phosphorylated signal was enhanced and the electrophoretic mobility was decreased when neurons were prelabeled in the presence of okadaic acid (OA) and MG132 (lane 6).

protein, extracts from fetal and adult brains were incubated in the presence of $\left[\gamma_{-}{ }^{32} \mathrm{P}\right]$ ATP followed by the immunoprecipitation of p35 and then analysis by SDS-PAGE (Fig. 4C). ${ }^{32}$ P-labeled p35 was detected using anti-p35 antibody (C-19) in immunoprecipitates from both fetal and adult brain extracts incubated at $30^{\circ} \mathrm{C}$ for $1 \mathrm{hr}$ (Fig. 4C, lanes 3 and 4 ). In addition, phosphorylated p35 was also immunoprecipitated from rat primary cultured neurons that were metabolically labeled with $\left[{ }^{32} \mathrm{P}\right]$ orthophosphate (Fig. $4 C$, lane 5). When cultured neurons were treated with both 0.1 $\mu \mathrm{M}$ okadaic acid and the proteasome inhibitor MG132 (50 $\mu \mathrm{M})$, the ${ }^{32} \mathrm{P}$ signal in $\mathrm{p} 35$ increased and the electrophoretic mobility of p35 decreased (Fig. 4C, lane 6). These results further indicate that p35 is a phosphoprotein.

\section{Phosphorylation of $\mathrm{p} 35$ by Cdk5 suppresses its cleavage to} p25 by calpain

p35 in adult cortical slices is susceptible to calpain cleavage (Fig. $1 B$ ). Because $\mathrm{p} 35$ is also less phosphorylated in adult tissue than in fetal tissue, we inferred that unphosphorylated p35 in adult brains might be more susceptible to calpain cleavage than is the phosphorylated p 35 in fetal brains. We examined whether the difference in susceptibility to calpain cleavage is derived from the p35 protein itself, using p35 immunoprecipitated from fetal or adult brain extracts. When immunoprecipitated p35 was incubated with purified calpain in the presence of $\mathrm{Ca}^{2+}, \mathrm{p} 35$ from fetal brain was more resistant to cleavage than p35 from adult brain (Fig. 5A).

The possibility that phosphorylation of $\mathrm{p} 35$ serves as a protec-

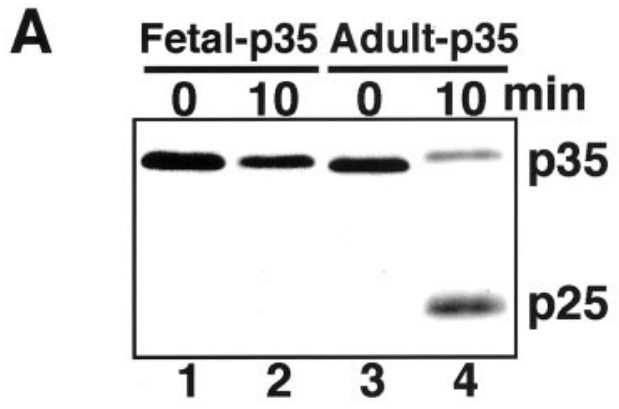

\section{Fetal brain extract}

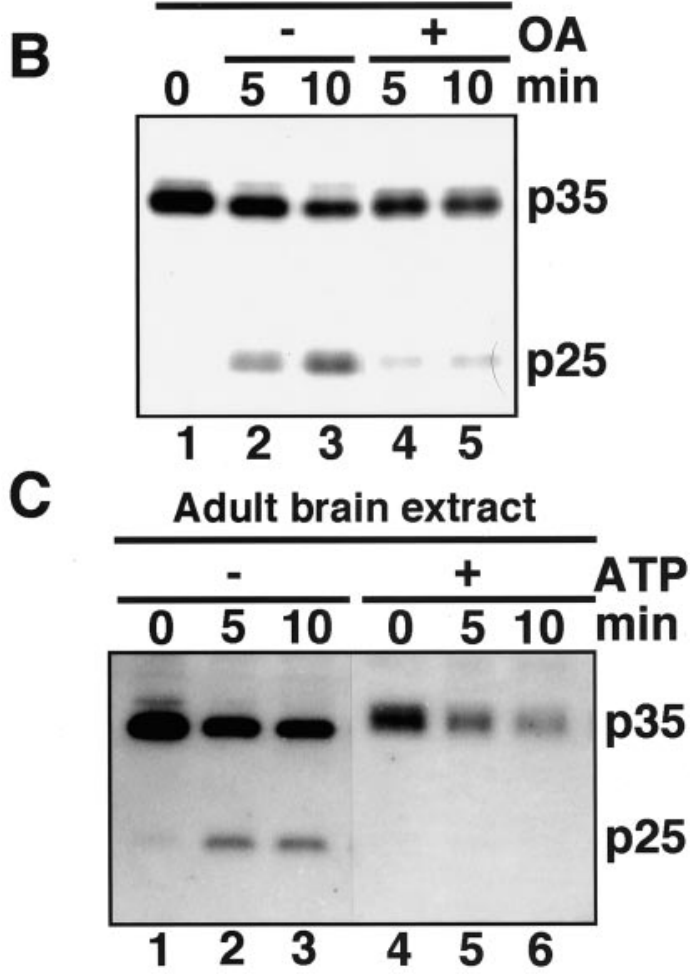

Figure 5. Phosphorylation of $\mathrm{p} 35$ blocked the cleavage of $\mathrm{p} 35$ to $\mathrm{p} 25$ in rat brain extracts. $A$, Adult p35 was more susceptible to calpain cleavage than fetal p35. p35 immunoprecipitated from fetal (lanes 1 and 2) or adult (lanes 3 and 4 ) brain extracts was treated with $\mathrm{m}$-calpain in the presence of $1 \mathrm{~mm} \mathrm{CaCl}$ for 0 min (lanes 1 and 3) or 10 min (lanes 2 and 4). p35 and p25 were detected by immunoblotting. $B, 0$ kadaic acid suppressed $\mathrm{Ca}^{2+}$-induced cleavage of $\mathrm{p} 35 \mathrm{in}$ fetal brain extracts. Fetal brain extracts were incubated with $5 \mathrm{~mm} \mathrm{CaCl}_{2}$ in the absence (lanes 2 and 3 ) or presence (lanes 4 and 5) of $10 \mu \mathrm{m}$ okadaic acid (OA) for $0 \mathrm{~min}$ (lane 1), 5 min (lanes 2 and 4), or $10 \mathrm{~min}$ (lanes 3 and 5). p35 and p25 were detected by immunoblotting. C, Adult brain extracts were incubated in the absence (lanes 1-3) or presence (lanes 4 - 6) of $1 \mathrm{~mm} \mathrm{Mg-ATP} \mathrm{for}$ $30 \mathrm{~min}$ and then incubated further in the presence of $5 \mathrm{~mm} \mathrm{CaCl}$ for $0 \mathrm{~min}$ (lanes 1 and 4 ), $5 \mathrm{~min}$ (lanes 2 and 5), or $10 \mathrm{~min}$ (lanes 3 and 6). p35 and p25 were detected by immunoblotting.

tive mechanism was tested. The addition of $\mathrm{Ca}^{2+}$ to fetal brain extracts resulted in the cleavage of p 35 to p25 (Fig. 5B, lanes 2 and 3 ), as observed previously with adult brain extract (Kusakawa et al., 2000). However, when the phosphorylation state of fetal brain p35 was maintained by the inhibition of protein phosphatases in the presence of $10 \mu \mathrm{M}$ okadaic acid (the concentration required to inhibit PP2B as well as PP1 and PP2A), $\mathrm{Ca}^{2+}$-dependent cleavage of p35 to p 25 was suppressed (Fig. 5B, lanes 4 and 5). We confirmed that calpain activity was unaffected by okadaic acid (data not shown). However, preincubation of adult brain extracts with ATP, which induces the phosphorylation of unphosphorylated adult $\mathrm{p} 35$, also suppressed $\mathrm{Ca}^{2+}$-dependent cleavage of $\mathrm{p} 35$ to 
A

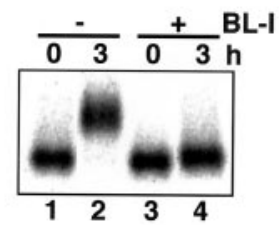

B
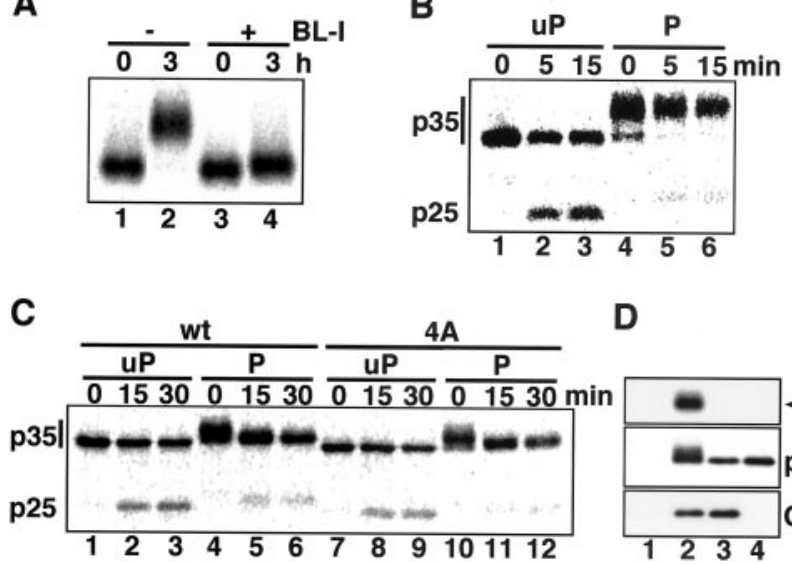

D

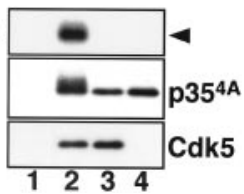

Figure 6. Phosphorylation of $\mathrm{p} 35$ by $(\mathrm{dk} 5$ blocked the cleavage to $\mathrm{p} 25$ by calpain. $A$, Inhibition of p35 phosphorylation by BL-I. ${ }^{35} \mathrm{~S}$-labeled $\mathrm{p} 35$ complexed with Cdk5 was incubated with $1 \mathrm{~mm} \mathrm{Mg}$-ATP at $30^{\circ} \mathrm{C}$ in the absence (lanes 1 and 2) or presence (lanes 3 and 4) of $50 \mu \mathrm{m}$ BL-I for $0 \mathrm{hr}$ (lanes 1 and 3) or $3 \mathrm{hr}$ (lanes 2 and 4). B, Phosphorylation-dependent suppression of p35 cleavage by calpain. $\mathrm{p} 35$ was phosphorylated by incubation with $1 \mathrm{~mm} \mathrm{Mg-ATP}$ at $30^{\circ} \mathrm{C}$ for $3 \mathrm{hr}$ as in $A$. Unphosphorylated ( $u P$; lanes $1-3$ ) or phosphorylated ( $P$; lanes $4-6)$ p35/Cdk5 was treated with $\mathrm{m}$-calpain in the presence of $1 \mathrm{~mm} \mathrm{CaCl}_{2}$ for $0 \mathrm{~min}$ (lanes 1 and 4 ), 5 min (lanes 2 and 5), or 15 min (lanes 3 and 6). C, Phosphorylation-dependent suppression of the cleavage of the $\mathrm{p} 35^{4 \mathrm{~A}}$ mutant by calpain. Unphosphorylated wild-type (wt) p35 (lanes 1-3), phosphorylated wild-type p35 (lanes 4-6), unphosphorylated p35 ${ }^{4 \mathrm{~A}}$ mutant (lanes 7-9), and phosphorylated $\mathrm{p} 35^{\mathrm{AA}}$ mutant (lanes 10-12) each were complexed with C $\mathrm{dk} 5$ and incubated in adult brain extract in the presence of $1 \mathrm{~mm} \mathrm{CaCl}$ for 0 min (lanes 1, 4, 7, and 10), 15 min (lanes 2, 5, 8, and 11), or 30 min (lanes 3, 6, 9, and 12). D, Phosphorylation of $\mathrm{p} 35^{4 \mathrm{~A}}$ by Cdk5. p35 $5^{4 \mathrm{~A}}$ complexed with wild-type Cdk5 (lane 2), kinase-dead Cdk5 (a Lys33Thr mutant; lane 3), or $\mathrm{p} 35^{4 \mathrm{~A}}$ alone (lane 4$)$ were incubated with $0.1 \mathrm{~mm}\left[\gamma^{-32}\right.$ P]ATP for $30 \mathrm{~min}$, and their phosphorylation was analyzed by imaging plate after SDS-PAGE. Lane 1 is a control in which a nickelbead-bound fraction of the uninfected insect cell lysate was applied. ${ }^{32} \mathrm{P}$ incorporation was detected only in $\mathrm{p} 35^{\mathrm{4A}}$ coinfected with wild-type Cdk5 (lane 2, arrowhead at top). A slight decrease in electrophoretic mobility of $\mathrm{p} 35^{4 \mathrm{~A}}$ was also observed (lane 2, middle). (dk5 is shown at the bottom.

p25 (Fig. 5C, lanes 5 and 6). Together, these results suggest that phosphorylation of p35 can serve to protect it from calpaindependent conversion to $\mathrm{p} 25$ in the brain extract.

An in vitro reconstitution approach was used to examine whether phosphorylation by $\mathrm{Cdk} 5$ can protect the cleavage of $\mathrm{p} 35$. $\left[{ }^{35} \mathrm{~S}\right]$ methionine-labeled $\mathrm{p} 35$ was synthesized by in vitro translation and then mixed with brain extract to allow the formation of a complex with Cdk5. The p35/Cdk5 complex then was immunoprecipitated with an antibody directed against Cdk5. When reconstituted p35/Cdk5 was incubated with ATP, the electrophoretic mobility of ${ }^{35}$ S-labeled p35 was decreased (Fig. $6 \mathrm{~A}$, lane 2), and this was suppressed by the Cdk5 inhibitor BL-I (Fig. 6A, lane 4). These results indicate that p35 is phosphorylated by Cdk5. After the addition of purified calpain and $1 \mathrm{~mm} \mathrm{CaCl}$, unphosphorylated $\mathrm{p} 35$ displayed substantial cleavage to p25 (Fig. 6B, lanes 2 and 3), whereas phosphorylated p35 did not (Fig. 6B, lanes 5 and 6 ). To identify the site(s) of phosphorylation by Cdk5, we generated a p35 mutant $\left(\mathrm{p} 35^{4 \mathrm{~A}}\right)$, in which the four (S/T)P sites $\left(\mathrm{Ser}^{8}\right.$, $\mathrm{Thr}^{138}$, $\mathrm{Ser}^{170}$, and $\left.\mathrm{Thr}^{197}\right)$ that constitute the minimum consensus phosphorylation sequences for Cdk5 were replaced with Ala. $\left[{ }^{35} \mathrm{~S}\right]$ methionine-labeled $\mathrm{p} 35^{4 \mathrm{~A}}$ was synthesized by in vitro translation and formed a complex with Cdk5 as described above. When incubated with ATP, however, $\mathrm{p} 35^{4 \mathrm{~A}}$ exhibited a decrease in electrophoretic mobility (Fig. 6C, lane 10), as observed for wild-type 35 (Fig. 6C, lane 4). The phosphorylation of $\mathrm{p} 35^{4 \mathrm{~A}}$ was shown by the Cdk5-dependent incorporation of ${ }^{32} \mathrm{P}$ into recombinant $\mathrm{p} 35^{4 \mathrm{~A}}$ with the decrease in electrophoretic mobility (Fig. $6 D$, lane 2). When $\mathrm{p} 35^{4 \mathrm{~A}}$ was phosphorylated, $\mathrm{p} 35^{4 \mathrm{~A}}$ showed
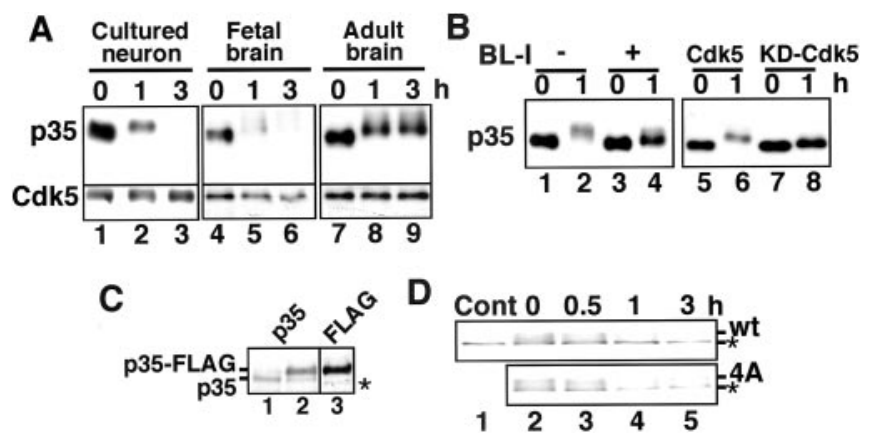

Figure 7. Phosphorylation of $\mathrm{p} 35$ by $\mathrm{Cdk} 5$ induced the degradation of $\mathrm{p} 35$ by proteasomes. $A$, Degradation of $\mathrm{p} 35$ was induced in cultured neuron extract and fetal brain extract in vitro but not in adult brain extract. Extracts prepared from cultured neurons (lanes 1-3) and fetal (lanes 4-6) and adult (lanes 7-9) brains were incubated with both $1 \mathrm{~mm}$ Mg-ATP and $10 \mu \mathrm{m}$ okadaic acid at $30^{\circ} \mathrm{C}$ for $0 \mathrm{hr}$ (lanes 1, 4, and 7), $1 \mathrm{hr}$ (lanes 2, 5, and 8), and $3 \mathrm{hr}$ (lanes 3, 6, and 9). $\mathrm{p35}$ and $\mathrm{Cdk} 5$ were detected by immunoblotting. B, Phosphorylation by $C \mathrm{dk} 5$ was required for the degradation of $\mathrm{p} 35$. Cultured neuron extract was incubated with $1 \mathrm{~mm} \mathrm{Mg-ATP}$ and $10 \mu \mathrm{M}$ okadaic acid in the absence (lanes 1 and 2) or presence (lanes 3 and 4) of $50 \mu \mathrm{m} \mathrm{BL-I}$ for $0 \mathrm{hr}$ (lanes 1 and 3) or $1 \mathrm{hr}$ (lanes 2 and 4). p35 complexed with Cdk5 (lanes 5 and 6 ) or kinase-dead Cdk5 (KD-Cdk5; a Lys33Thr mutant; lanes 7 and 8), which was expressed in Sf9 cells, was incubated with $1 \mathrm{~mm} \mathrm{Mg}$-ATP and $10 \mu \mathrm{m}$ okadaic acid for $0 \mathrm{hr}$ (lanes 5 and 7 ) or $1 \mathrm{hr}$ (Ianes 6 and 8). p35 was detected by immunoblotting. C, Expression of FLAG-tagged p35 in cultured neurons. Construction and infection of adenovirus carrying FLAG-tagged p35 are described in Materials and Methods. p35 was detected in uninfected (lane 1) or infected (lanes 2 and 3) cultured neurons by anti-p35 (lanes 1 and 2) or anti-FLAG (lane 3) antibodies. The asterisk denotes a nonspecific band. D, Degradation of $\mathrm{p} 35$ or mutant $\mathrm{p} 35^{\mathrm{AA}}$ in cultured neurons. Cultured neurons infected with an adenovirus carrying the FLAG-tagged wild-type $\mathrm{p} 35$ ( $w t$; top) or the $\mathrm{p} 35^{\mathrm{AA}}$ mutant $(4 A$; bottom) were treated with CHX for $0,0.5,1$, or $3 \mathrm{hr}$, and $\mathrm{p} 35$ was detected by immunoblotting with anti-FLAG antibody. Uninfected control neurons are designated Cont. The asterisk indicates a nonspecific band.

resistance to calpain as strongly as p $35^{\mathrm{wt}}$ (Fig. 6C, lanes 10-12). These results suggest that phosphorylation at a non-(S/T)P site(s) in p35 may protect $\mathrm{p} 35$ from cleavage by calpain to $\mathrm{p} 25$.

\section{Phosphorylation of $\mathrm{p} 35$ by Cdk5 stimulates the degradation of $\mathrm{p} 35$ by proteasomes}

Previous results indicate that $\mathrm{p} 35$ from fetal brain slice cultures has a faster rate of turnover than $\mathrm{p} 35$ derived from adult brain (Fig. $1 B$ ). Other results indicate that $\mathrm{p} 35$ from fetal tissue is more phosphorylated (Fig. 3). To demonstrate the effects of phosphorylation on the proteasomal degradation of $\mathrm{p} 35$, extracts prepared from cultured cortical neurons or fetal brains were incubated in the presence of both ATP and okadaic acid. Addition of ATP and okadaic acid to extracts of cultured neurons induced hyperphosphorylation of $\mathrm{p} 35$, as demonstrated by an increase in its electrophoretic mobility (Fig. 7A, lane 2). Longer periods of incubation were accompanied by a decrease in $\mathrm{p} 35$ levels (Fig. $7 \mathrm{~A}$, lane 3 ) caused by proteasomal degradation, as reported previously (Saito et al., 1998). Similar degradation was observed in fetal brain extracts (Fig. 7A, lanes 5 and 6). In contrast, the addition of both ATP and okadaic acid to extracts from adult brains caused an increase in electrophoretic mobility, but no subsequent degradation of p35 was observed (Fig. 7A, lanes 8 and 9). These results suggest that phosphorylation of $\mathrm{p} 35$, although protecting it from calpain cleavage, facilitates the proteasomal degradation that is probably responsible for the high turnover rate of p35 in fetal tissue. However, in adult tissue, where $\mathrm{p} 35$ exists in an unphosphorylated state, phosphorylation was induced in vitro but did not appear to facilitate proteasomal degradation. Total levels of Cdk5 in the extracts were unaffected by any of the treatments (Fig. 7A, bottom). 
The phosphorylation that facilitated proteasomal degradation was also Cdk5-dependent phosphorylation. Inhibition of Cdk5 activity by BL-I appeared to slow the rate of degradation (Fig. $7 B$, lanes 2 and 4 ). This was confirmed further by an experiment using kinase-dead recombinant Cdk5 (Cdk5 $\left.{ }^{\mathrm{KD}}\right)$. Wild-type recombinant Cdk5 (Cdk5 ${ }^{\mathrm{WT}}$ ) complexed with p35 was shown to behave similarly to endogenous p35/Cdk5. p35 in the recombinant complex of $\mathrm{p} 35 / \mathrm{Cdk} 5^{\mathrm{WT}}$ was phosphorylated and degraded by incubation with cultured neuron extract in the presence of ATP and okadaic acid (Fig. 7B, lane 6). In contrast, p35 complexed with $C d k 5^{\mathrm{KD}}$ did not undergo degradation, although a slight increase in electrophoretic mobility was observed (Fig. $7 B$, lane 8). The (S/T)P-phosphorylation sites in p35 are involved in proteasomal degradation in COS7 cells (Patrick et al., 1998). We tested whether this is also the case in neurons by infecting adenoviruses that express wild-type $\mathrm{p} 35^{\mathrm{WT}}$-FLAG or the $\mathrm{p} 35^{4 \mathrm{~A}}$-FLAG mutant into embryonic cultured neurons. p35 ${ }^{\mathrm{WT}}$-FLAG was detected at a slightly higher molecular weight than endogenous p35 by immunoblotting with anti-p35 (Fig. 7C, lane 2) and antiFLAG antibodies (Fig. 7C, lane 3). There was a decrease in endogenous p35 in neurons expressing p35 ${ }^{\mathrm{WT}}$-FLAG (Fig. 7C, lane 2). The lower-molecular-weight band detected with anti-FLAG antibody (Fig. 7C, lane 3, asterisk) was a nonspecific product that also was detected in nontransfected cell extract (Fig. 7D, Cont). The turnover rates of $\mathrm{p} 35^{\mathrm{WT}}$-FLAG and $\mathrm{p} 35^{4 \mathrm{~A}}$-FLAG were estimated in the presence of CHX by immunoblotting with antiFLAG antibody (Fig. 7D). p35 ${ }^{4 \mathrm{~A}}$-FLAG was detected $3 \mathrm{hr}$ after the addition of CHX (Fig. $7 D, 4 A$ in bottom), whereas p35 ${ }^{\mathrm{WT}}$ FLAG was degraded (Fig. 7D, WT in top). These results indicate that Cdk5-dependent phosphorylation at the $(\mathrm{S} / \mathrm{T}) \mathrm{P}$ sites of $\mathrm{p} 35$ also predisposes it to degradation by proteasomes in neurons.

\section{Phosphorylation of p35 does not affect the kinase activity of Cdk5}

Despite the observation that p35 was autophosphorylated by Cdk5, p35 in adult brains was unphosphorylated (Fig. 3). This suggested the possibility that Cdk5 complexed with adult unphosphorylated p35 is less active. To address this possibility, the kinase activity of Cdk5 complexed with phosphorylated and unphosphorylated p35 was measured. Phosphorylated p35/Cdk5 and unphosphorylated p35/Cdk5 complexes were prepared by immunoprecipitation from fetal and adult brain extracts incubated with or without ATP, and their kinase activities were assayed using histone $\mathrm{H} 1$ or the microtubule-associated protein tau as substrates (Fig. 8). To maintain the phosphorylation state during immunoprecipitation, phosphatase inhibitors were added to the solutions, and the phosphorylation states were confirmed after immunoprecipitation by immunoblotting (data not shown). There were no clear differences in the ability of Cdk5 to phosphorylate either histone $\mathrm{H} 1$ or tau when associated with phosphorylated or unphosphorylated forms of $\mathrm{p} 35$. These data indicate that the phosphorylation status of p35 has no apparent effect on Cdk5 activity.

\section{Discussion}

We have shown in this study for the first time that p35 is phosphorylated in fetal rat brains and that the phosphorylation level of p35 decreases during postnatal brain development, resulting in the unphosphorylated form of p35 in the adult brain, which is easily cleaved to p25 in brain slices and extracts when calpain is activated. When p35 was phosphorylated, the cleavage of p35 to p25 was suppressed. The critical role of phosphorylation in proteasome-dependent degradation also was demonstrated in

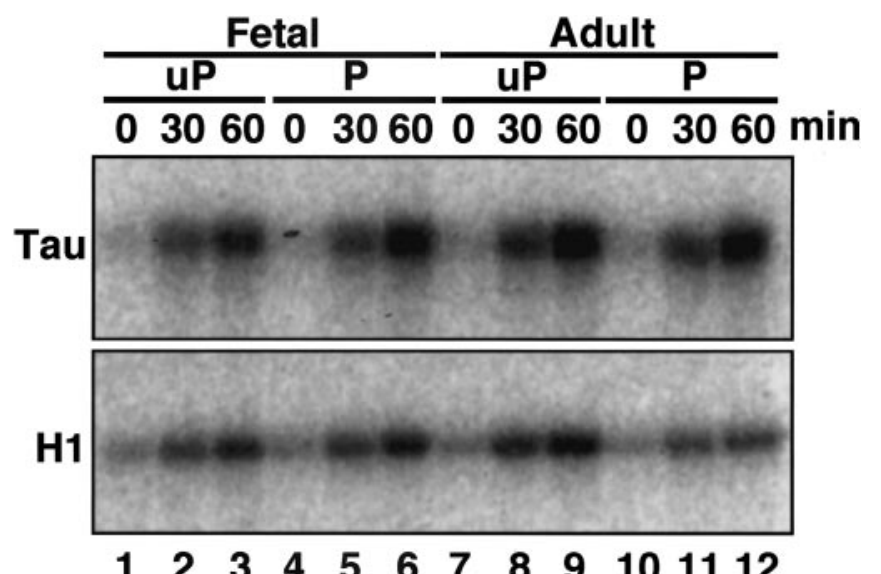

Figure 8. Effect of $\mathrm{p} 35$ phosphorylation on Cdk5 kinase activity. Cdk5 bound to unphosphorylated ( $u P$; lanes $1-3$ and $7-9$ ) or phosphorylated ( $P$; lanes $4-6$ and $10-12$ ) p35 was prepared from fetal and adult brain extracts as described in Materials and Methods. Kinase activity of the complexes was assayed at $30^{\circ} \mathrm{C}$ for 0 min (lanes $1,4,7$, and 10 ), 30 min (lanes 2, 5, 8, and 11 ), and 60 min (lanes 3, 6, 9, and 12) using tau (Tau; top) or histone H1 (H1; bottom) as substrate.

brain extracts and in cultured neurons transfected with an unphosphorylatable mutant of $\mathrm{p} 35$. These results suggest that neurons in the fetal rat brain are equipped with a mechanism that protects $\mathrm{p} 35$ from unfavorable cleavage to $\mathrm{p} 25$, and that the increased susceptibility to cleavage that is acquired by p35 in the adult rat brain might be relevant to the vulnerability of mature neurons.

p35 is reported to be phosphorylated by Cdk5 in vitro (Lew et al., 1994; Tsai et al., 1994; Saito et al., 1998). However, its role and even its in vitro occurrence have not been shown previously. We have shown by metabolic labeling with $\left[{ }^{32} \mathrm{P}\right]$ orthophosphate that p35 is phosphorylated in cultured neurons. Phosphorylation of p35 in rat brains was indicated by phosphorylation-dependent electrophoretic mobility changes. It is interesting to note that the phosphorylation status of p35 changes with postnatal rat brain development; p35 is phosphorylated in fetal brains and unphosphorylated in adult brains. Phosphorylation was the result of phosphorylation by Cdk5. Recombinant p35 complexed with $\mathrm{Cdk} 5$ was phosphorylated only by incubation with ATP, but the replacement of Cdk5 with a kinase-dead mutant of Cdk5 failed to phosphorylate p35, even in brain extract. We constructed a p35 mutant $\left(\mathrm{p} 35^{4 \mathrm{~A}}\right.$ ) whose all four SP or TP minimum consensus phosphorylation sites for C $\mathrm{dk} 5$ were changed to Ala and examined whether the phosphorylation sites are present within these four sites and, if so, whether the phosphorylation affects the proteolytic patterns. $\mathrm{p} 35^{4 \mathrm{~A}}$ was still phosphorylated. The phosphorylation changed the proteasomal degradation property but did not change the cleavage property of $\mathrm{p} 35$. The simple interpretation is that the phosphorylation involved in preventing calpain cleavage occurs at a Ser or Thr residue other than the (S/T)P motif. At present, however, we cannot exclude possibilities of other modifications or protein factors that regulate the proteolysis of $\mathrm{p} 35$.

The phosphorylation of $\mathrm{p} 35$ has at least two roles in the proteolysis of p35: the prevention of calpain-dependent cleavage and the stimulation of proteasome-dependent degradation. As a consequence of the changes in phosphorylation status with development, the proteolytic properties of p35 also change with brain maturation. However, we do not know at present why the phosphorylation status of p 35 changes with development despite autophosphorylation. Because Cdk5 displayed 
similar kinase activity regardless of whether p35 was phosphorylated, there may be some other factors controlling the phosphorylation state, and therefore the proteolytic properties, of p35 in neurons.

It has been suggested that the cleavage of p35 to p25 is associated with neuronal cell death (Kusakawa et al., 2000; Lee et al., 2000). The cleavage of $\mathrm{p} 35$ releases active Cdk5 from localized compartments in the vicinity of the membranes or cytoskeleton, thereby allowing its free movement in the cytoplasm (Patrick et al., 1999; Kusakawa et al., 2000) and probably causing the abnormal phosphorylation of otherwise unphosphorylated proteins (Patrick et al., 1999). Although this issue must be examined further in the future, it is compatible with the hypothesis that the activation of cell cycle-promoting factors in postmitotic neurons induces cell death (Evans et al., 1995). This generalized concept could be tested easily on the particular properties of p35, because p35 is a fragile protein that is easily cleaved. For example, p35 is immediately cleaved to $\mathrm{p} 25$ by passage through a purification column (Kusakawa et al., 2000) or during short-term postmortem delay (Taniguchi et al., 2001; Yoo and Lubec, 2001). Cdk5 complexed with p35 has not been purified from brain tissues successfully (Ishiguro et al., 1992b; Lew et al., 1992; Hisanaga et al., 1993). However, only a limited amount of p25 is detected in freshly and carefully prepared rat brains, although calpain should be activated in living neurons, for example, during excitation or local injury (Carafoli and Molinari, 1998). The difference in the generation of p25 in vitro and in vivo may suggest some mechanism(s) that prevents the involvement of aberrant Cdk5 activity in neuronal cell death, even when calpain is activated locally and transiently. Phosphorylation of $\mathrm{p} 35$ could be one such protective mechanism in fetal brain neurons.

Rapid turnover could be another mechanism reducing the uncontrolled conversion of p35 to p25. Although the physiological importance of the rapid turnover of p35 is not understood, the faster the rate of $\mathrm{p} 35$ turnover the less chance there is for it to be cleaved. It has been suggested that the turnover rate (i.e., the rate of proteasomal degradation) is regulated by phosphorylation of p35 (Patrick et al., 1998; Saito et al., 1998). In this study, we have confirmed that phosphorylation stimulates the degradation of p35 in cultured neurons and brain extracts. Unexpectedly, the degradation of p35 also changed developmentally. Whereas p35 in fetal brain slices and extract was degraded rapidly, p35 in both adult brain slices and extracts was not. Because proteasomal activity itself does not differ much between fetal and adult brain extracts (data not shown), ubiquitination of $\mathrm{p} 35$, phosphorylation of $\mathrm{p} 35$, or both may be reduced in the adult rat brain. We think that the phosphorylation of $\mathrm{p} 35$ may be a more important determinant for the half-life of $\mathrm{p} 35$, although it is possible that the degradation system for $\mathrm{p} 35$, which is not known at all, is also downregulated. Why the phosphorylation of $\mathrm{p} 35$ decreases in the adult brain is a question to be addressed in the future.

The phosphorylation that may prevent calpain-dependent cleavage occurs constitutively in fetal brains and cultured neurons. This phosphorylation was maintained in fetal brain extracts by incubating only with either ATP or okadaic acid. The phosphorylation induced in the adult brain extracts by incubation with ATP occurred at the site involved in preventing calpain cleavage but not the site facilitating proteasomal degradation (our unpublished observation). Additional phosphorylation was detected transiently during the process of degradation by proteasomes and was detected stably only when neurons were treated with both okadaic acid and MG132. The latter phosphorylation may be a signal for the proteasomal degradation of p35. The site(s) occurs within the four SP or TP proline-directed kinase consensus sites $\left(\mathrm{Ser}^{8}, \mathrm{Thr}^{138}, \mathrm{Thr}^{170}\right.$, and $\mathrm{Ser}^{197}$ ) in $\mathrm{p} 35$. This observation is consistent with the observation of Patrick et al. (1998) in transfected COS cells. It was suggested that the phosphorylation site that may relate to calpain cleavage was different from the site related to proteasomal degradation. The former site, which has yet to be identified, is now under investigation.

Decreased phosphorylation decreased the turnover rate of p 35 and increased the propensity for calpain cleavage in adult brains, which, in turn, would increase the chance of p35 cleavage after calpain activation. Because unphosphorylated adult p35 is more resistant to proteasomal degradation than phosphorylated fetal p35, the longer half-life of p35 may be more important than the protection of p35 from calpain-dependent cleavage in adult brains. This age-dependent susceptibility to calpain may be related to age-dependent neuronal cell death in neurodegenerative diseases. An accumulation of p25 has been reported in Alzheimer's brains (Patrick et al., 1999) and in the brains of the amyotrophic lateral sclerosis mouse model (Nguyen et al., 2001). However, cleavage also is induced during postmortem delay in normal rat and human brains (Taniguchi et al., 2001; Yoo and Lubec, 2001). Therefore, the involvement of p $25 / \mathrm{Cdk} 5$ in neurodegenerative disease should be investigated. Understanding the cellular mechanism that regulates $\mathrm{p} 25 / \mathrm{Cdk} 5$ production in neurons also would provide information on the involvement of Cdk5 in pathogenesis.

\section{References}

Baumann K, Mandelkow EM, Biernat J, Piwnica-Worms H, Mandelkow E (1993) Abnormal Alzheimer-like phosphorylation of tau-protein by cyclin-dependent kinases cdk2 and cdk5. FEBS Lett 336:417-424.

Carafoli E, Molinari M (1998) Calpain: a protease in search of a function? Biochem Biophys Res Commun 247:193-203.

Chae T, Kwon YT, Bronson R, Dikkes P, Li E, Tsai LH (1997) Mice lacking p35, a neuronal specific activator of Cdk5, display cortical lamination defects, seizures, and adult lethality. Neuron 18:29-42.

Evans GI, Brown I, Whyte M, Harrington E (1995) Apoptosis and the cell cycle. Curr Opin Cell Biol 7:825-834.

Hisanaga S, Ishiguro K, Uchida T, Okumura E, Okano T, Kishimoto T (1993) Tau protein kinase II has a similar characteristic to cdc2 kinase for phosphorylating neurofilament proteins. J Biol Chem 268:15056-15060.

Ishiguro K, Omori A, Takamatsu M, Sato K, Arioka M, Uchida T, Imahori K (1992a) Phosphorylation sites on tau by tau protein kinase I, a bovine derived kinase generating an epitope of paired helical filaments. Neurosci Lett 148:202-206.

Ishiguro K, Takamatsu M, Tomizawa K, Omori A, Takahashi M, Arioka M, Uchida T, Imahori K (1992b) Tau protein kinase I converts normal tau protein into A68-like component of paired helical filaments. J Biol Chem 267:10897-10901.

Kusakawa G, Saito T, Onuki R, Ishiguro K, Kishimoto T, Hisanaga S (2000) Calpain-dependent proteolytic cleavage of the p35 cyclin-dependent kinase 5 activator to p25. J Biol Chem 275:17166-17172.

Laemmli UK (1970) Cleavage of structural proteins during the assembly of the head of bacteriophage T4. Nature 227:680-685.

Lee K-Y, Rosales JL, Tang D, Wang JH (1996) Interaction of cyclindependent kinase 5 (Cdk5) and neuronal Cdk5 activator in bovine brain. J Biol Chem 271:1538-1543.

Lee MS, Kwon YT, Li M, Peng J, Friedlander RM, Tsai LH (2000) Neurotoxicity induces cleavage of p35 to p25 by calpain. Nature 405:360-364.

Lew J, Winkfein RJ, Paudel HK, Wang JH (1992) Brain proline-directed protein kinase is a neurofilament kinase which displays high sequence homology to p34cdc2. J Biol Chem 267:25922-25926.

Lew J, Huang QQ, Qi Z, Winkfein RJ, Aebersold R, Hunt T, Wang JH (1994) A brain-specific activator of cyclin-dependent kinase 5. Nature 371:423-426.

Nguyen MD, Lariviere RC, Julien JP (2001) Deregulation of Cdk5 in a mouse model of ALS: toxicity alleviated by perikaryal neurofilament inclusions. Neuron 30:135-147. 
Nikolic M, Dudek H, Kwon YT, Ramos YF, Tsai LH (1996) The cdk5/p35 kinase is essential for neurite outgrowth during neuronal differentiation. Genes Dev 10:816-825.

Ohshima T, Ward JM, Huh CG, Longenecker G, Veeranna, Pant HC, Brady RO, Martin LJ, Kulkarni AB (1996) Targeted disruption of the cyclindependent kinase 5 gene results in abnormal corticogenesis, neuronal pathology and perinatal death. Proc Natl Acad Sci USA 93:11173-11178.

Patrick GN, Zhou P, Kwon YT, Howley PM, Tsai LH (1998) p35, the neuronalspecific activator of cyclin-dependent kinase 5 (Cdk5) is degraded by the ubiquitin-proteasome pathway. J Biol Chem 273:24057-24064.

Patrick GN, Zukerberg L, Nikolic M, de la Monte S, Dikkes P, Tsai LH (1999) Conversion of p 35 to p 25 deregulates Cdk5 activity and promotes neurodegeneration. Nature 402:615-622.

Ploug M, Jensen AL, Barkholt V (1989) Determination of amino acid compositions and $\mathrm{NH}_{2}$-terminal sequences of peptides electroblotted onto PVDF membranes from tricine-sodium dodecyl sulfate-polyacrylamide gel electrophoresis: application to peptide mapping of human complement component C3. Anal Biochem 181:33-39.

Poon RY, Lew J, Hunter T (1997) Identification of functional domains in the neuronal Cdk5 activator protein. J Biol Chem 272:5703-5708.

Saido TC, Yokota M, Nagao S, Yamaura I, Tani E, Tsuchiya T, Suzuki K, Kawashima S (1993) Spatial resolution of fodrin proteolysis in postischemic brain. J Biol Chem 268:25239-25243.

Saito T, Ishiguro K, Onuki R, Nagai Y, Kishimoto T, Hisanaga S (1998)
Okadaic acid-stimulated degradation of p35, an activator of CDK5, by proteasome in cultured neurons. Biochem Biophys Res Commun 252:775-778.

Schagger H, von Jagow G (1987) Tricine-sodium dodecyl sulfate-polyacrylamide gel electrophoresis for the separation of proteins in the range from 1 to $100 \mathrm{kDa}$. Anal Biochem 166:368-379.

Taniguchi S, Fujita Y, Hayashi S, Kakita A, Takahashi H, Murayama S, Saido TC, Hisanaga S, Iwatsubo T, Hasegawa M (2001) Calpain-mediated degradation of p35 to p25 in postmortem human and rat brains. FEBS Lett 489:46-50.

Tokuoka H, Saito T, Yorifuji H, Wei F, Kishimoto T, Hisanaga S (2000) Brainderived neurotrophic factor-induced phosphorylation of neurofilament- $\mathrm{H}$ subunit in primary cultures of embryo rat cortical neurons. J Cell Sci 113:1059-1068.

Tsai LH, Delalle I, Caviness Jr VS, Chae T, Harlow E (1994) p35 is a neuralspecific regulatory subunit of cyclin-dependent kinase 5. Nature 371:419-423.

Uchida T, Ishiguro K, Ohnuma J, Takamatsu M, Yonekura S, Imahori K (1994) Precursor of cdk5 activator, the $23 \mathrm{kDa}$ subunit of tau protein kinase II: its sequence and developmental change in brain. FEBS Lett 355:35-40.

Wang KK (2000) Calpain and caspase: can you tell the difference? Trends Neurosci 23:20-26.

Yoo BC, Lubec G (2001) p25 protein in neurodegeneration. Nature 411: 763-764. 